Published in The Geneva Risk and Insurance Review, 2018, Volume 43, Issue 1, pp 77-94, which should be cited to refer to this work.

DOI : $10.1057 /$ s10713-018-0029-8

\title{
ON THE PROPERTIES OF HIGH-ORDER NON-MONETARY MEASURES FOR RISKS
}

\author{
Christophe Courbage \\ Geneva School of Business Administration \\ University of Applied Sciences Western Swizterland (HES-SO) \\ Henri Loubergé \\ University of Geneva \\ Geneva School of Economics and Management, Swiss Finance Institute \\ Béatrice Rey \\ Université de Lyon, Lyon 69007, France \\ CNRS, GATE Lyon Saint-Etienne, Ecully 69130, France

\begin{abstract}
This paper investigates how welfare losses for facing high-order risk increases change when the risk environment of the decision maker is altered. To that aim, we define the $n$ th-order utility premium as a measure of pain associated with facing the passage of one risk to a more severe one and we examine some of its properties. Changes in risk are expressed through the concept of stochastic dominance of order $n$. The paper investigates more particularly welfare changes of merging increases in risk, first ignoring background risks, then taking them into account. Merging increases in risk may be beneficial or not, depending on whether background risks are considered and how. The paper also provides conditions on individual preferences for superadditivity of the $n$ th-order utility premium. The results conrm the importance and usefulness of two analytical concepts: mixed risk aversion and risk apportionment.
\end{abstract}

Keywords: mixed risk aversion, risk apportionment, merging increases in risk, superadditivity, $n$ thorder utility premium

JEL classication: D81 


\section{INTRODUCTION}

The issue of how the presence of multiple risks modify individual behaviour in the face of another risk has been leading to a prolific literature during the last decades. ${ }^{1}$ Most of these studies use monetary measures to analyze behaviour towards risk, the most well-known being the Arrow-Pratt risk premium ${ }^{2}$ and the willingness to pay (Pratt, 1988). More recently, a few papers have used non-monetary measures to provide new behavioural results in the face of risks. In particular, the concept of utility premium originally introduced by Friedman and Savage (1948) has regained interest. For instance, Eeckhoudt and Schlesinger (2006) rely on the utility premium to propose a unified approach explaining the meaning of the signs of the successive derivatives of the utility function. Eeckhoudt and Schlesinger (2009) also reexamine the properties of the utility premium and explain the relevance of this tool for decision-making. Menegatti (2011) examines the relationship between the risk premium and the utility premium and showed that both measures do not overlap: the risk premium can be a mistaken measure of the reduction in utility caused by risk. Starting from the utility premium, Crainich and Eeckhoudt (2008) and Courbage and Rey (2010) turn to higher orders by introducing non-monetary measures of prudence and temperance. Such non-monetary measures not only offer alternative tools to analyze the individual loss of welfare due to the presence of risks, but also allow for much simpler conditions on individual preferences to predict behaviour towards risks. ${ }^{3}$

An issue of great importance when dealing with measures of risks is how these measures react to a riskier environment. In particular, knowing how welfare losses of facing increases in risk change as a function of the number of risk exposures offers crucial knowledge on how individuals react to riskier environment. Our objective is to address these issues, starting from very general definitions of increases in risk and of loss of welfare. For this reason, the paper defines the " $n$ th-order utility premium" as a measure of pain associated with facing the passage from one risk to a worse one, with changes in risk expressed through the concept of stochastic dominance of order $n$.

Our $n$ th-order utility premium is not new. It was used before, without being given a name, in Eeckhoudt et al. (2009) in their proof of Theorem 3 and in Ebert et al. (2017)

\footnotetext{
${ }^{1}$ See Eeckhoudt and Gollier (2013) for a review.

${ }^{2}$ Arrow (1970), Pratt (1964).

${ }^{3}$ Note, however, that a well-known deficiency of the utility premium compared to the risk premium is its inadequacy for interpersonal comparisons. This is due to the fact that the utility premium is not unique under linear transformations of the utility function. To circumvent this difficulty, Crainich and Eeckhoudt (2008) introduced the monetary utility premium - the utility premium divided by the marginal utility. Li and Liu (2014) and Huang and Stapleton (2015) have used the monetary utility premium to derive comparative risk aversion results. In this paper we do not address interpersonal comparisons of loss of welfare.
} 
in their proof of Theorem 2. But we define it here explicitly and our main objective in this paper is to derive some of its key properties.

Motivated by the work of Eeckhoudt and Gollier (2001) on the properties of monetary measures for risk, we first address the issue of whether the pain for facing multiple risk changes is greater or less than the sum of the pains for facing each risk change independently. This issue should not be confused with the concept of mutual aggravation of risk changes developed in Ebert et al. (2017). In their work, an individual faces two risks that are deteriorating and the issue is whether facing the two changes in risk simultaneously is more painful than the sum of the pains for facing each change in risk one by one (see their equation (1)). They obtain that this is indeed the case. There is mutual aggravation of risk changes (Theorem 2), and even greater mutual aggravation when the risk changes are more severe (Theorem 3). In our case, we want to focus on a mathematical property of our $n$ th-order utility premium and we consider two different situations where an individual exposed to one risk faces an aggravation of this risk. The question arises: is the non-monetary measure of the two risk changes together greater or less than the sum of the monetary measures of each individual risk change ${ }^{4}$ ? In economic life, this question arises when the benefits obtained for merging independent entities (or firms) are considered. More specifically, we examine the following question: knowing that separate entities will be subject to high-order risk increases, is it beneficial for the owner of these entities to merge them or not? It turns out that mixed risk aversion, as defined by Caballé and Pomansky (1996), is key to the answer. ${ }^{5}$

This question is addressed in two steps. In the first step, the entities are considered as entirely separated, i.e. managed independently, with the consequence that each of them is unaffected by the risk level of the other. In this case, it turns out that merging the changes in risk is not beneficial under mixed risk aversion. In a second step, we recognize that the decision maker is the owner of both entities, which leads to introduce a link between them. The risk of each entity is now a background risk for the other entity. We obtain that, in this case, merging the risks may be beneficial if the risk situation of each entity is transparent: the definition of background risk takes into account, for each entity, the increase in risk for the other entity.

These results on risk merging under conditions of changes in risk are new. But they may easily be related to other literature in the economics of risk. The result in the absence of background risk implies a result for a related but different issue, the superadditivity or subadditivity of the $n$ th-order utility premium. A measure is said to be superadditive or convex in the number of risks if the measured value of two risks is superior to the sum of the values of each risk; the opposite holding for subadditivity. Superadditivity/subadditivity

\footnotetext{
${ }^{4}$ See our equation (16) below.

${ }^{5}$ This is not unexpected, given the role of mixed risk aversion in the link between expected utility and $n$ th-order stochastic dominance. See, e.g., Theorem 1 in Eeckhoudt et al. (2009). Mixed risk aversion is also required to obtain the results of mutual aggravation in Ebert et al. (2017).
} 
sheds light on whether risks are self-aggravating for individuals. For instance, Eeckhoudt and Gollier (2001) show that risk vulnerability (see Gollier and Pratt, 1996) is a sufficient condition for superadditivity of the risk premium. The concept of subadditivity of risk measures has also become popular by the concept of coherent risk measures defined by Artzner et al. (1999). A (monetary) measure is said to be coherent if it satisfies four axioms, among which subadditivity. In our case, the paper shows that the $n$ th-order utility premium is superadditive under mixed risk aversion.

The results in presence of background risk bring us closer to the question examined by Ebert et al. (2017). Indeed, starting from a different angle, we reproduce their result of mutual aggravation. But not necessarily so. Depending on how background risk is defined, one can obtain either mutual aggravation or mutual mitigation of risk changes. The two results offer reinterpretations of the preference for combining "good" with "bad" introduced by Eeckhoudt et al. (2009). In the same manner, we also rely on this latter paper to show under which conditions the $n$ th-order utility premium increases when the decision-maker's initial wealth becomes riskier. This result provides another reinterpretation of the preference for combining "good" with "bad", while generalizing Courbage and Rey (2010).

The paper is organised as follows. Section 2 introduces the benchmark model for non-monetary measures of risk and in particular the $n$ th-order utility premium. Section 3 presents our main results. It addresses the conditions on individual preferences for welfare changes due to risk merging. It also provides the answer to the question of whether the $n$ th-order utility premium is superadditive or subadditive. Section 4 investigates the effects of background risk, first by examining the impact of a riskier initial wealth on the $n$ th-order utility premium, then by introducing background risk in the evaluation of risk merging. Under appropriate conditions, it also provides the link with the work of Ebert et al. (2017). Finally, Section 5 offers a short conclusion.

\section{THE BENCHMARK MODEL}

\subsection{Non-monetary measures in the face of risks}

Non-monetary measures in the face of risks stem from the work of Friedman and Savage (1948) who used expected utility theory to define risk aversion and introduced two ways for its measure. The two measures reflect the subjective cost of risk for a risk averter.

Let an individual's final wealth be represented by $x+\tilde{\epsilon}$ where $x(x>0)$ denotes the initial wealth of the individual and $\tilde{\epsilon}$ is a zero-mean random variable. ${ }^{6}$ The first measure of risk aversion in the face of the risk $\tilde{\epsilon}$ at wealth level $x$ is a monetary measure, the risk

\footnotetext{
${ }^{6} \mathrm{We}$ assume that the support of $\tilde{\epsilon}$ is defined such that $x+\epsilon$ is in the domain of $u$.
} 
premium $\pi(x, \tilde{\epsilon})$, and is such that:

$$
E[u(x+\widetilde{\epsilon})]=u(x-\pi(x, \tilde{\epsilon}))
$$

where $u$ denotes the individual's von Neumann-Morgenstern utility function (with $u^{\prime}(x) \geq$ $0 \forall x)$ and $E$ denotes the expectation operator. $\pi(x, \tilde{\epsilon})$ is the amount of money that the agent is ready to pay to get rid of the zero-mean risk $\widetilde{\epsilon} . \pi(x, \tilde{\epsilon}) \geq 0$ if and only if the individual is risk-averse $\left(u^{\prime \prime}(x) \leq 0 \forall x\right)$.

The second one is a non-monetary measure of risk aversion, the utility premium, $w_{A}(x, \tilde{\epsilon}):$

$$
w_{A}(x, \widetilde{\varepsilon})=u(x)-E[u(x+\widetilde{\varepsilon})] .
$$

$w_{A}(x, \widetilde{\varepsilon})$ measures the degree of "pain" associated with facing the risk $\widetilde{\varepsilon}$, where pain is measured by the loss in expected utility from adding the risk $\widetilde{\varepsilon}$ to wealth $x$. From Jensen's inequality, $w_{A}(x) \geq 0$ if and only if $u^{\prime \prime}(x) \leq 0 \forall x .^{7}$

More recently additional non-monetary measures were introduced, based on the definitions of higher-order risk attitudes, prudence and temperance. Prudence $\left(u^{\prime \prime \prime} \geq 0\right)$ first introduced by Kimball (1990) in a saving context is also known as risk apportionment of order 3 in the sense of Eeckhoudt and Schlesinger (2006). These authors show that for a prudent individual:

$$
\frac{1}{2} u(x-l)+\frac{1}{2} E[u(x+\widetilde{\varepsilon})] \geq \frac{1}{2} u(x)+\frac{1}{2} E[u(x-l+\widetilde{\varepsilon})] .
$$

According to Eeckhoudt and Schlesinger's terminology, a prudent individual prefers the left-hand side term of Eq. (3) to the right-hand side term because the pains, the sure loss $-l(l>0)$ and the zero-mean risk $\widetilde{\varepsilon}$, are better apportioned in the left-hand side term than in the right-hand side term. In the left-hand side term the pains are disaggregated while they are aggregated (concentrated in a single state of nature) on the right hand side term. The prudence utility premium as introduced by Crainich and Eeckhoudt (2008), denoted $w_{P}(x, \widetilde{\varepsilon})$, measures the increase in pain of facing the risk $\widetilde{\varepsilon}$ in the presence of a sure loss $l>0$. This is defined as follows:

$$
w_{P}(x, \widetilde{\varepsilon})=u(x-l)-E[u(x-l+\widetilde{\varepsilon})]-(u(x)-E[u(x+\widetilde{\varepsilon})]),
$$

which is equivalent to:

$$
w_{P}(x, \widetilde{\varepsilon})=w_{A}(x-l, \tilde{\epsilon})-w_{A}(x, \tilde{\epsilon}) .
$$

Naturally, $w_{P}(x, \widetilde{\varepsilon}) \geq 0$ if and only if $u^{\prime \prime \prime} \geq 0$.

Temperance $\left(u^{\prime \prime \prime \prime} \leq 0\right)$, first introduced by Kimball (1992) in a context of risk management in the presence of background risk, is known as risk apportionment of order 4 in

\footnotetext{
${ }^{7}$ We assume throughout this article that the utility function $u$ is $n$-times differentiable. As usual, we assume that the derivative of order $k(\forall k \geq 1)$, denoted $u^{(k)}(x)$, has a constant sign in the domain of $u$ : $u^{(k)}(x) \geq 0$ or $u^{(k)}(x) \leq 0 \forall x$.
} 
the sense of Eeckhoudt and Schlesinger (2006). It reflects a preference for disaggregation of two independent zero-mean risks $\tilde{\theta}$ and $\tilde{\epsilon}$. Eeckhoudt and Schlesinger (2006) show that for a temperant individual:

$$
\frac{1}{2} E[u(x+\tilde{\theta})]+\frac{1}{2} E[u(x+\widetilde{\varepsilon})] \geq \frac{1}{2} u(x)+\frac{1}{2} E[u(x-l+\tilde{\theta}+\widetilde{\varepsilon})]
$$

A temperant individual prefers the left-hand side term of Eq. (6) to the right-hand side term because the pains are better apportioned. The temperance utility premium as introduced by Courbage and Rey $(2010)$, denoted $w_{T}(x, \widetilde{\varepsilon})$, measures the increase in pain of facing the risk $\widetilde{\varepsilon}$ in the presence of an independent zero-mean risk $\tilde{\theta}$. It writes as follows:

$$
w_{T}(x, \widetilde{\varepsilon})=E[u(x+\tilde{\theta})]-E[u(x+\tilde{\theta}+\widetilde{\varepsilon})]-(u(x)-E[u(x+\widetilde{\varepsilon})]),
$$

which is equivalent to:

$$
w_{T}(x, \widetilde{\varepsilon})=w_{A}(x+\tilde{\theta}, \tilde{\epsilon})-w_{A}(x, \tilde{\epsilon}) .
$$

$w_{T}(x, \widetilde{\varepsilon}) \geq 0$ if and only if $u^{(4)} \leq 0$.

Courbage and Rey (2010) suggested an extension of these measures to higher orders defining the utility premium by iteration following Eeckhoudt and Schlesinger (2006). Denoting $w_{(2)}(x, \widetilde{\varepsilon})$ the Friedman and Savage (1948) utility premium of Eq. (2), we can proceed from their remark by defining for all $n$ even and $n \geq 2$ :

$$
w_{(n+1)}(x, \tilde{\epsilon})=w_{(n)}(x-l, \tilde{\epsilon})-w_{(n)}(x, \tilde{\epsilon})
$$

with $l>0$ and

$$
w_{(n+2)}(x, \tilde{\epsilon})=w_{(n)}\left(x+\tilde{\theta}_{n}, \tilde{\epsilon}\right)-w_{(n)}(x, \tilde{\epsilon}),
$$

where $\tilde{\theta}_{n}$ is an independent random variable (i.e. random variables $\tilde{\epsilon}, \tilde{\theta}_{2}, \tilde{\theta}_{4}, \tilde{\theta}_{6}$, etc, are mutually independent) and such that $E\left(\tilde{\theta}_{n}\right)=0$. As an illustration, when $n=2$, $w_{(n+1)}(x, \tilde{\epsilon})$ corresponds to the prudence utility premium, $w_{P}(x, \tilde{\epsilon})$, and $w_{(n+2)}(x, \tilde{\epsilon})$ corresponds to the temperance utility premium, $w_{T}(x, \tilde{\epsilon})$, as defined by Eqs. (4) and (7).

\subsection{The $n$ th-order utility premium}

While Courbage and Rey (2010) suggested to define utility premia of higher orders by iteration of the previous utility premia defined in a context of specific lotteries, our objective in this paper is to introduce a very general way to define the utility premium at higher orders and to disclose its properties. Our definition uses the concept of stochastic dominance of order $n$.

Let's consider two risky situations: a first situation represented by the random variable $Y$ and a second one represented by the random variable $X$. We assume that $X$ and $Y$ are independent, and that $Y$ dominates $X$ via $n$ th-order stochastic dominance $\left(X \preceq_{n-S D} Y\right)$. 
The concept of $n$ th-order stochastic dominance is defined as follows. ${ }^{8}$ Consider $Y$ and $X$ with $F$ and $G$, respectively, their two cumulative distribution functions of wealth, defined over a probability support contained within the interval $[a, b]$. Define $F_{1}=F$ and $G_{1}=G$. Now define $F_{k+1}(z)=\int_{a}^{z} F_{k}(t) d t$ and $G_{k+1}(z)=\int_{a}^{z} G_{k}(t) d t$ for $k \geq 1$. The variable $Y$ dominates $X$ via $n$ th-order stochastic dominance $\left(X \preceq_{n-S D} Y\right)$ if $F_{n}(z) \leq$ $G_{n}(z)$ for all $z$, and if $F_{k}(b) \leq G_{k}(b)$ for $k=1,2, . ., n$.

When the $n-1$ moments of $X$ and $Y$ are equal, $n$ th-order stochastic dominance coincides with Ekern's (1980) concept of increase in $n$ th-order risk ( $X \preceq_{n} Y$ ). Ekern's (1980) definition includes the case of mean-preserving increase in risk of Rothshild and Stiglitz (1970) as well as the case of increase in downside risk defined by Menezes et al. (1980). These cases represent, respectively, a second-degree and a third-degree increase in risk.

We want to define the non-monetary measure of the cost of facing the risk transition, i.e., the passage from $Y$ to $X$ where $Y$ dominates $X$ via $n$ th-order stochastic dominance $\left(X \preceq_{n-S D} Y\right)$. Let's define the function $w$ as follows: ${ }^{9}$

$$
w(x ; Y, X)=E[u(x+Y)]-E[u(x+X)] .
$$

The function $w(x ; Y, X)$ measures the degree of pain associated with facing the passage from the risk $Y$ to the less favorable one, $X$, when the decision-maker's initial wealth is $x$. We formulate the following definition.

Definition. Given two independent risks, $Y$ and $X$ such that $Y$ dominates $X$ via nth-order stochastic dominance $\left(X \preceq_{n-S D} Y\right)$, the function $w$ defined as $w(x ; Y, X)=$ $E[u(x+Y)]-E[u(x+X)]$ is named the "nth-order utility premium"10. It measures the degree of pain due to the aggravating nth-order stochastic dominance risk.

We first observe that, from Ingersoll (1987), $w(x ; Y, X) \geq 0$ for all $x$ if and only if $(-1)^{(1+k)} u^{(k)} \geq 0 \forall k=1, \ldots, n$. Note that $(-1)^{(1+k)} u^{(k)} \geq 0 \forall k \geq 1$ means that all odd derivatives of $u$ are positive and all even derivatives of $u$ are negative. Following Brockett and Golden (1987) and according to Caballé and Pomansky (1996), an individual with such a utility function is said to be mixed risk averse. Hence, for all order $n$, the $n$ th-order utility premium of a mixed risk averse agent is always positive. In other words, such an individual always incurs a pain when facing the passage from the risk $Y$ to a less favorable

\footnotetext{
${ }^{8}$ See for example Jean $(1980,1984)$ or Ingersoll (1987).

${ }^{9} \mathrm{We}$ assume throughout this article that the support of any random variable $\tilde{z}$ is defined such that $x+z$ is in the domain of $u$.

${ }^{10}$ The $n$ th-order utility premium was already used by Eeckhoudt et al. (2009) as well as by Ebert et al. (2017) without being given a name. However, Harris Schlesinger referred to this utility premium as the "comparative utility premium" during a presentation of an earlier version of Ebert et al. (2017) at the 2013 EGRIE seminar in Paris.
} 
one $X$ dominated via $n$ th-order stochastic dominance. If the utility function $u$ satisfies $(-1)^{(1+k)} u^{(k)} \geq 0 \forall k=1, \ldots, n$, we will label $u$ as mixed risk averse from order 1 to $n$.

An important particular case of the $n$ th-order utility premium corresponds to the case where, using the terminology introduced by Eeckhoudt et al. (2009), $Y$ and $X$ are lotteries respectively combining: "good" with "bad" in both states; and "good with good" in one state and "bad with bad" in the other state. Obviously, the terminology introduced by Eeckhoudt et al. (2009) is very close to the concept of risk apportionment in Eeckhoudt and Schlesinger (2006). Eeckhoudt and Schlesinger (2006) defined risk apportionment of order $n(n \geq 1)$, by imposing preferences over simple lotteries ${ }^{11}$. Risk apportionment of order $n$ is equivalent to the condition $\operatorname{sign}\left\{u^{(n)}\right\}=(-1)^{(n+1)}$ which can be interpreted as a preference for harms disaggregation at order $n$. These higher-order risk attitudes entail a preference for combining relatively good outcomes with bad ones and can be interpreted as a desire to disaggregate the harms of unavoidable risks and losses ${ }^{12}$. Eeckhoudt et al. (2009) generalized Eeckhoudt and Schlesinger (2006) and established that a decision maker exhibiting risk apportionment prefers not to group the two relatively "bad" lotteries in the same state, where "bad" is defined via higher-order stochastic dominance. Such a decision maker prefers combining "good" with "bad" in each state of nature. They derived in particular the following theorem:

Theorem [Eeckhoudt, Schlesinger and Tsetlin, 2009] Suppose that $Y_{i}$ dominates $X_{i}$ via $n_{i}$ th-order stochastic dominance for $i=1,2$ and suppose that $X_{1}, X_{2}, Y_{1}, Y_{2}$ are mutually independent risks. The 50-50 lottery $\left[X_{1}+Y_{2}, Y_{1}+X_{2}\right]$ dominates the 50-50 lottery $\left[X_{1}+X_{2}, Y_{1}+Y_{2}\right]$ via $\left(n_{1}+n_{2}\right)$ th-order stochastic dominance.

Thus, in their work, our $Y$ and $X$ above may be represented by the following 50-50 lotteries

$$
Y=\left[X_{1}+Y_{2}, Y_{1}+X_{2}\right] \text { and } X=\left[X_{1}+X_{2}, Y_{1}+Y_{2}\right]
$$

where $n_{1}+n_{2}=n$. The basic idea is that a decision maker with a utility function $u$ such that $(-1)^{(1+k)} u^{(k)} \geq 0 \forall k=1, \ldots, n$ will allocate the state-contingent risks in such a way as not to group the two "bad" risks in the same state, where "bad" is defined via $i$ th-order stochastic dominance. Such an individual prefers the 50-50 lottery $\left[X_{1}+Y_{2}, Y_{1}+X_{2}\right]$ to the 50-50 lottery $\left[X_{1}+X_{2}, Y_{1}+Y_{2}\right]$ :

$$
\frac{1}{2} E\left[u\left(X_{1}+Y_{2}\right)\right]+\frac{1}{2} E\left[u\left(Y_{1}+X_{2}\right)\right] \geq \frac{1}{2} E\left[u\left(X_{1}+X_{2}\right)\right]+\frac{1}{2} E\left[u\left(Y_{1}+Y_{2}\right)\right] .
$$

The passage from $Y$ to $X$ creates a loss of utility due to the aggregation of the harms. In such a case, our $n$ th-order utility premium measures the degree of pain due to the

\footnotetext{
${ }^{11}$ These lotteries were characterized by Roger (2011) who established that they only differ by their moments of order greater than or equal to $n$. See also Ebert (2013).

${ }^{12}$ See also the experimental results obtained by Deck and Schlesinger $(2010,2014)$.
} 
passage from $Y$ to $X$.

Other particular cases of the $n$ th-order utility premium are the various premia defined in the previous sub-section for which $Y$ dominates $X$ via $n$ th-order Ekern's dominance ( $X \preceq_{n} Y$ ). Indeed, following Ekern (1980), if $X \preceq_{n} Y$ then $w(x ; Y, X) \geq 0$ for all $x$ if and only if $(-1)^{(1+n)} u^{(n)} \geq 0$. For instance, when $Y=0$ and $X$ is a zero-mean background risk, $X=\tilde{\epsilon}$ with $E(\tilde{\epsilon})=0$, the function $w$ writes as $w(x ; 0, \tilde{\epsilon})=u(x)-E[u(x+\tilde{\epsilon})]=w_{A}(x, \widetilde{\varepsilon})$. This is the 2nd-order utility premium introduced by Friedman and Savage (1948) to define the non-monetary risk aversion measure: $w(x ; 0, \tilde{\epsilon}) \geq 0$ if and only if the individual is risk averse $\left(u^{\prime \prime} \leq 0\right)$. When $Y$ and $X$ are defined as equiprobable lotteries, they are particular cases of lotteries defined by Eq. (10). For instance, when $Y$ and $X$ are defined as equiprobable lotteries describing an increase in downside risk (Menezes et al. (1980)): $Y=[-l, \tilde{\epsilon}]$ and $X=[0,-l+\tilde{\epsilon}]$ i.e. $Y_{1}=0, Y_{2}=0, X_{1}=-l$ and $X_{2}=\tilde{\epsilon}$, the function $w$ writes as

$$
w(x ; Y, X)=\frac{1}{2} w_{P}(x, \tilde{\epsilon}),
$$

where $w_{P}(x, \tilde{\epsilon})$ is the prudence utility premium defined by Crainich and Eeckhoudt (2008). Here $w(x ; Y, X)$ is the 3rd-order utility premium. It measures the degree of pain due to the passage from $Y$ to $X$ with $X \preceq_{3} Y$. When $Y$ and $X$ are defined as the following

equiprobable lotteries: $Y=[\tilde{\theta}, \tilde{\epsilon}]$ and $X=[0, \tilde{\theta}+\tilde{\epsilon}]$ where $\tilde{\theta}$ and $\tilde{\epsilon}$ are independent and zero mean random variables, i.e. , $Y_{1}=0, Y_{2}=0, X_{1}=\tilde{\theta}$ and $X_{2}=\tilde{\epsilon}$, the function $w$ writes as

$$
w(x ; Y, X)=\frac{1}{2} w_{T}(x, \tilde{\epsilon}),
$$

where $w_{T}(x, \tilde{\epsilon})$ is the temperance utility premium defined by Courbage and Rey (2010). Here $w(x ; Y, X)$ is the 4th-order utility premium. It measures the degree of pain due to the passage from $Y$ to $X$ with $X \preceq_{4} Y$.

\section{MERGING CHANGES IN RISKS AND THE SUPERADDITIVITY OF THE $n$ th-ORDER UTILITY PREMIUM}

Ebert et al. (2017) offered a reinterpretation of Eeckhoudt et al.'s (2009) results expressed in Eq. (11) by showing that mixed risk aversion ensures mutual aggravation of risk changes i.e. the decision maker's trait of perceiving two risk changes as mutually aggravating. They rewrote Eq. (11) equivalently as:

$$
\begin{aligned}
E\left[u\left(Y_{1}+Y_{2}\right)\right]-E\left[u\left(X_{1}+X_{2}\right)\right] \geq\left(E \left[u \left(Y_{1}+\right.\right.\right. & \left.\left.\left.Y_{2}\right)\right]-E\left[u\left(X_{1}+Y_{2}\right)\right]\right) \\
& +\left(E\left[u\left(Y_{1}+Y_{2}\right)\right]-E\left[u\left(Y_{1}+X_{2}\right)\right]\right)
\end{aligned}
$$

i.e., the utility from avoiding both risk changes at once is greater than the utility from avoiding the first risk change (passage from $Y_{1}$ to $X_{1}$ ) plus the the utility from avoiding 
the second risk change (passage from $Y_{2}$ to $X_{2}$ ). According to Ebert et al. (2017), mutual aggravation means that experiencing risks increases one at a time is comparatively better than having to face two risk increases at once. This is useful for risk management applications, in particular, as shown in the web appendix to their paper. Using the $n$ th-order utility premium, Eq. (14) can be rewritten $\mathrm{as}^{13}$ :

$$
w\left(x ; Y_{1}+Y_{2}, X_{1}+X_{2}\right) \geq w\left(x ; Y_{1}+Y_{2}, X_{1}+Y_{2}\right)+w\left(x ; Y_{2}+Y_{1}, X_{2}+Y_{1}\right) .
$$

In this section, we consider a related but different issue. We consider a change in the risk environment of the decision-maker that corresponds to the process of merging changes in risks. More specifically, we consider a change in risk defined as the passage from $Y_{1}$ to $X_{1}$ and from $Y_{2}$ to $X_{2}$ with $X_{1} \preceq_{n_{1}-S D} Y_{1}$ and $X_{2} \preceq_{n_{2}-S D} Y_{2}$ where risks $Y_{1}, Y_{2}, X_{1}$ and $X_{2}$ are mutually independent and where $Y_{1} \preceq_{2-S D} 0$ and $Y_{2} \preceq_{2-S D} 0$. We wonder under which conditions on the utility function $u$ the non-monetary cost of the total change in risk (passage from $\left(Y_{1}+Y_{2}\right)$ to $\left.\left(X_{1}+X_{2}\right)\right)$ is larger than the sum of the non-monetary costs of each change in risk (passage from $Y_{1}$ to $X_{1}$ and independently passage from $Y_{2}$ to $X_{2}$ ). More formally, we wonder what properties of $u$ ensure the following inequality:

$$
w\left(x ; Y_{1}+Y_{2}, X_{1}+X_{2}\right) \geq w\left(x ; Y_{1}, X_{1}\right)+w\left(x ; Y_{2}, X_{2}\right)
$$

If $Y_{1}$ and $Y_{2}$ are both dominated by zero at order 2 , the following proposition provides conditions for such a comparison (see proof in the appendix).

Proposition 1. Consider mutually independent random variables $X_{1}, X_{2}, Y_{1}$ and $Y_{2}$, such that $X_{1} \preceq_{n_{1}-S D} Y_{1}, X_{2} \preceq_{n_{2}-S D} Y_{2}, Y_{1} \preceq_{2-S D} 0$ and $Y_{2} \preceq_{2-S D} 0$. Then the $n$ th-order utility premium satisfies $w\left(x ; Y_{1}+Y_{2}, X_{1}+X_{2}\right) \geq w\left(x ; Y_{1}, X_{1}\right)+w\left(x ; Y_{2}, X_{2}\right)$ for all mixed risk-averse utility functions $u$ from order 1 to $n_{1}+n_{2}, \forall n_{1} \geq 2, \forall n_{2} \geq 2$.

Proposition 1 means that welfare is reduced by merging changes in risks instead of facing them separately, i.e., the welfare loss of both increases in risks taken together is larger than the sum of welfare losses from assuming each increase in risk separately.

A possible illustration of this result is given by the current changes in risk faced by many financial institutions. They must take into account the entry into the market of digitally-powered financial platforms developed by GAFA (Google, Apple, Facebook, Amazon) and others. A bank with settlement in different regions faces a change in risk for every local entity, with the changes in risk being specific to the competition environment of each region. Is it beneficial for this bank to merge the different settlements in one single entity to face the changes in risk? Proposition 1 tells us that this is not the case if the

\footnotetext{
${ }^{13}$ Note that both Theorem 2 in Ebert et al. (2017) and our Proposition 1 below assume mixed risk aversion. But their theorem uses Liu's (2014) theorem, a more general condition, while we use stochastic dominance. We also assume that $Y_{1}$ and $Y_{2}$ are dominated by zero at order 2 .
} 
decision process of the bank may be represented by the preferences of a mixed risk-averse decision maker.

Proposition 1 also implies the superadditivity of the $n$ th-order utility premium. ${ }^{14}$ From the risk theory literature (see for example Bühlmann (1985) or Gerber and Goovaerts (1981)), it is well-known that financial risks are very often self-aggravating. This would suggest that the cost of risk for two independent risks should be greater than the sum of costs of the two risks taken in isolation. If it were the case, the cost of risk would be superadditive. The definition of superadditivity is the following. A real-valued function $f$ is superadditive if $f\left(z_{1}+z_{2}\right)$ is larger than $f\left(z_{1}\right)+f\left(z_{2}\right)$ for all $z_{1}>0$ and $z_{2}>0$. The opposite inequality holding true for subadditivity.

Here, we address the issue of superadditivity when the cost of risk is defined in nonmonetary terms through the concept of the $n$ th-order utility premium. We consider the passage from a risky situation $Y$ to a less favorable one $X_{i}(i=1,2)$. The superadditivity of the $n$ th-order utility premium writes as

$$
w\left(x ; Y, X_{1}+X_{2}\right) \geq w\left(x ; Y, X_{1}\right)+w\left(x ; Y, X_{2}\right),
$$

where $Y, X_{1}$ and $X_{2}$ are mutually independent random variables. Subadditivity of the $n$ th-order utility premium corresponds naturally to the opposite inequality. Starting from Proposition 1 we obtain the following result (see proof in the appendix).

Corollary 1.Consider mutually independent random variables $X_{1}, X_{2}$ and $Y$, such that $X_{1} \preceq_{n_{1}-S D} Y, X_{2} \preceq_{n_{2}-S D} Y$, with $Y \preceq_{2-S D} 0$. Then the nth-order utility premium is superadditive (i.e. $w\left(x ; Y, X_{1}+X_{2}\right) \geq w\left(x ; Y, X_{1}\right)+w\left(x ; Y, X_{2}\right)$ ) for all mixed risk-averse utility functions $u$ from order 1 to $n_{1}+n_{2}, \forall n_{1} \geq 2, \forall n_{2} \geq 2$.

According to Corollary 1, the pain of facing a change in two risks simultaneously is higher than the sum of the pains of facing the two changes in risk separately for all mixed risk-averse decision makers.

In the specific case of the Friedman-Savage utility premium, with $X_{1}$ and $X_{2}$ independent zero-mean risks, Corollary 1 means that the pain of facing two risks simultaneously is higher than the sum of the pains of facing each risk separately for a risk-averse and temperant individual. Eeckhoudt and Gollier (2001) examine this issue when the cost of risk is defined in terms of risk premium. They show that the risk premium is superadditive if risk preferences are risk vulnerable. ${ }^{15}$ Hence, while risk vulnerability is required for monetary measures of risk to be superadditive, for which temperance is only a nec-

\footnotetext{
${ }^{14}$ We thank a referee for pointing out the link between the two properties.

${ }^{15}$ Risk vulnerability means that risk aversion increases with the presence of an independent background risk (Gollier and Pratt, 1996). Sufficient and necessary conditions on the utility function to have risk vulnerability are quite complex. A necessary condition for risk vulnerability is $u^{(4)} \leq 0$.
} 
essary condition, temperance here is sufficient to obtain superaditivity in the case of the Friedman-Savage utility premium.

We can illustrate the result of Corollary 1 in the spirit of Samuelson (1963) who points out that risk averters prefer to subdivide risks instead of facing them in one shot.

Let's consider three periods, period 1, period 2 and period 3. In period 1, the agent faces a risk $Y$. He knows that in the two other periods, instead of facing $Y$, he will face two other risks, $X_{1}$ and $X_{2}$, respectively which are both more severe than $Y$. For example, $X_{1}$ reflects the risk of a heavy medical treatment and $X_{2}$ represents the risk of a new job, while $Y$ is the status quo. The agent can decide in which period he will face the changes in risk. He can decide to face them one by one, in two consecutive periods, or to face them simultaneously in period 3 while maintaining the status quo in period 2 .

Ignoring the discount factor, the intertemporal expected utility writes in case 1 as $E u(x+Y)+E u\left(x+X_{1}\right)+E u\left(x+X_{2}\right)$ and in case 2 as $E u(x+Y)+E u(x+Y)+$ $E u\left(x+X_{1}+X_{2}\right)$. Saying that the intertemporal expected utility in case 2 is lower than the intertemporal expected utility in case 1 is equivalent to Eq. (17). Therefore mixed risk aversion ensures that the agent prefers to face the changes in risk one by one in consecutive periods 2 and 3 .

\section{OTHER PROPERTIES OF THE $n$ th-ORDER UTILITY PREMIUM}

In this section we consider the impact of background risk on the level of the $n$ thorder utility premium. The introduction of background risk means that the analysis is transposed to a multiple risk setting. Thus, we enter into the domain of issues addressed by Eeckhoudt et al. (2009) and Ebert et al. (2017). All our results are expressed as corollaries of the main theorem in Eeckhoudt et al. (2009). The novelty is that they are formulated as properties of the $n$ th-order utility premium. In addition, we also obtain that mutual mitigation of risks is possible, instead of mutual aggravation, depending on how background risk is introduced.

\subsection{The $n$ th-order utility premium and increases in background risk}

Following Courbage and Rey (2010), we can investigate, in the more general context of the $n$ th-order utility premium, how this measure reacts to the introduction on wealth of a sure loss $(-l$ with $l>0)$ or a zero-mean background risk $(\tilde{\epsilon}$ with $E(\tilde{\epsilon})=0)$. As intuition suggests, the pain increases in both cases under usual conditions on the signs of higher-order derivatives of the utility function. Indeed, considering the impact of a sure loss and a background risk on the $n$ th-order utility premium, we obtain:

$$
w(x-l ; Y, X)-w(x ; Y, X) \geq 0 \Leftrightarrow(-1)^{(k+1)} u^{(k)} \geq 0 \forall k=1, \ldots, n+1 .
$$




$$
w(x+\tilde{\epsilon} ; Y, X)-w(x ; Y, X) \geq 0 \Leftrightarrow(-1)^{(k+1)} u^{(k)} \geq 0 \forall k=1, \ldots, n+2 .
$$

These results are obtained by expanding the two inequalities and using Eeckhoudt et al. (2009), as explicitly demonstrated in Ebert et al. (2017). Eqs. (18) and (19) have intuitive explanations. If we consider a decision maker with a mixed risk-averse utility function from order 1 to $n+2$, Eq. (18) means that the $N$ th-order utility premium $(\forall N \leq n+1)$ is vulnerable to a sure loss in the sense that it increases with the introduction of a sure loss. Similarly, Eq. (19) means that the $N$ th-order utility premium $(\forall N \leq n)$ is vulnerable to a zero-mean background risk, i.e., it increases with the introduction of a zero-mean background risk.

Such analysis can be extended to a more general context by investigating the degree of pain associated with facing the passage from $Y$ to $X$ when the wealth level becomes riskier. A riskier wealth corresponds to the random wealth level, initially equal to $x+Y_{2}$, becoming $x+X_{2}$ where $Y_{2}$ dominates $X_{2}$ via $n_{2}$ th-order stochastic dominance $\left(X_{2} \preceq_{n_{2}-S D} Y_{2}\right.$ with $X_{2}$ and $Y_{2}$ being independent random variables). The degree of pain associated with facing the passage from $Y$ to $X$ when the wealth level becomes riskier is defined by the following expression:

$$
w\left(x+X_{2} ; Y, X\right)-w\left(x+Y_{2} ; Y, X\right) .
$$

A positive sign of (20) means that the pain of facing the passage from $Y$ to $X$ increases when the wealth level becomes riskier. We obtain the following result as a corollary of Eeckhoudt et al's (2009) theorem (see proof in the appendix).

Corollary 2. Consider mutually independent random variables $X, Y, X_{2}$, and $Y_{2}$, such that $X \preceq_{n_{1}-S D} Y$ and $X_{2} \preceq_{n_{2}-S D} Y_{2}$. Then $w\left(x+X_{2} ; Y, X\right)-w\left(x+Y_{2} ; Y, X\right) \geq 0$ for all mixed risk-averse utility functions $u$ from order 1 to $n_{1}+n_{2}$.

Corollary 2 can be interpreted in a similar way as Eqs. (18) and (19). If we consider a decision maker with a mixed risk-averse utility function from order 1 to $n+n_{2}$, it means that the $N$ th-order utility premium $\left(\forall N \leq n-n_{2}\right)$ is vulnerable to a detrimental change of order $n_{2}$ in the background risk. Corollary 2 generalizes results in Courbage and Rey (2010) using results on stochastic dominance and offers a reinterpretation of Eeckhoudt et al.'s (2009) result.

If we restrict our attention to the special case of Ekern dominance as often done in the literature, then, according to Ekern (1980), $w(x ; Y, X) \geq 0$ if and only if $(-1)^{(1+n)} u^{(n)} \geq 0$, i.e., risk apportionment of order $n$ holds $^{16}$. From Corollary 2, we can then extrapolate that the pain due to misaportionment of order $\left(n-n_{2}\right)$ is vulnerable to an increase in

\footnotetext{
${ }^{16}$ Note, however, that if risk apportionment of order $n$ holds, recent work by Menegatti (2015) shows that risk apportionment of order $j$ will hold, for $j=2, \ldots, n-1$, under very general conditions on the utility function. We are thus brought back to the condition of Corollary 2, even when Ekern increases in risk of order $n$ are considered, instead of stochastic dominance of order $n$.
} 
risk of order $n_{2}$. This offers an alternative interpretation of the sign of the $n$ th-order derivative of the utility function $\left(u^{(n)}\right)$ which can be easily understood and remembered, without reference to any specific decision problem. For instance, in the case of the utility premium for which $Y=0$ and $X=\tilde{\epsilon}\left(X \preceq_{2} Y\right)$, the degree of pain of facing the risk $\tilde{\epsilon}$ increases when initial wealth becomes risky (i.e., when any zero-mean risk $X_{2}$ is added to initial wealth) for a temperant individual. In the same way, in the case of the prudence premium, for which $X$ represents an increase in downside risk over $Y\left(X \preceq_{3} Y\right.$ ), the degree of pain of facing an increase in downside risk increases when initial wealth becomes risky for an individual featuring edginess ${ }^{17}$.

\subsection{Merging changes in risk under background risk}

In the context described by Proposition 1, the two risks sets $\left(Y_{1}, X_{1}\right)$ and $\left(Y_{2}, X_{2}\right)$ are considered in isolation. Nevertheless, these two risk sets are present in the decisionmaker's environment. A change of perspective arises if we take this fact into account, and if we consider that risk $2\left(X_{2}\right.$ or $\left.Y_{2}\right)$, is a background risk for the management of risk $1\left(X_{1}\right.$ or $\left.Y_{1}\right)$, and vice-versa. More specifically, we wonder under which conditions on the utility function $u$ the non-monetary cost of the total change in risk (passage from $\left(Y_{1}+Y_{2}\right)$ to $\left.\left(X_{1}+X_{2}\right)\right)$ is larger than the sum of the non-monetary costs of each change in risk taking account the global decision maker's environment, i.e., the passage from $Y_{1}$ to $X_{1}$ considering that risk 2 is a background risk for the management of risk 1 , and the passage from $Y_{2}$ to $X_{2}$ considering that risk 1 is a background risk for the management of risk 2. Interestingly, it turns out that this change of perspective can reverse the results. We obtain the following result as a corollary of Eeckhoudt et al.'s (2009) theorem.

Corollary 3. Consider mutually independent random variables $X_{1}, X_{2}, Y_{1}$, and $Y_{2}$ such that $X_{1} \preceq_{n_{1}-S D} Y_{1}$ and $X_{2} \preceq_{n_{2}-S D} Y_{2}$. Then the two following items (a) and (b) hold for all mixed risk-averse utility functions $u$ from order 1 to $n_{1}+n_{2}$ :

(a) $w\left(x ; Y_{1}+Y_{2}, X_{1}+X_{2}\right) \geq w\left(x+Y_{2} ; Y_{1}, X_{1}\right)+w\left(x+Y_{1} ; Y_{2}, X_{2}\right)$

(b) $w\left(x ; Y_{1}+Y_{2}, X_{1}+X_{2}\right) \leq w\left(x+X_{2} ; Y_{1}, X_{1}\right)+w\left(x+X_{1} ; Y_{2}, X_{2}\right)$.

The difference between items $(a)$ and $(b)$ arises from background risk considerations on the right-hand side. In item $(b)$, the background risks taken into account are the worse risks $X_{1}$ and $X_{2}$ while they are the better ones $Y_{1}$ and $Y_{2}$ in item $(a)$. In item $(b)$, the decision maker is aware that risk $Y_{2}$ will be replaced by risk $X_{2}$ when she feels the loss of welfare from facing the risk $X_{1}$ instead of $Y_{1}$. In item $(a)$, the decision maker is blind to this risk substitution. She feels a reduced loss from the sum of individual risk

\footnotetext{
${ }^{17}$ The concept of edginess, i.e. $u^{(5)} \geq 0$, was introduced by Lajeri-Chaherli (2004) to explain the effects of background risks on precautionary savings.
} 
substitutions because she ignores that risk $Y_{2}$ will be replaced by risk $X_{2}$ when dealing with risk 1, and she ignores that risk $Y_{1}$ will be replaced by risk $X_{1}$ when dealing with risk 2. In this sense, item $(b)$ reflects a kind of rational expectations, whereas item $(a)$ reflects blindness.

It should also be stressed that following Ebert et al. (2017), item (a) of Corollary 3 can have another interpretation in terms of mutual aggravation of risks changes. Using the perspective of these authors, we show that item (b) can also be interpreted in terms of mutual mitigation of risks changes. To reach such conclusion, we have to consider the following property of the $n$ th-order utility premium. Assume three mutually independent risks, $X, Y$ and $Z$, then $w(x+Z ; Y, X)=w(x ; Y+Z, X+Z)^{18}$. Using this property, Corollary 3 can be equivalently rewritten as follows.

Corollary 4. Consider mutually independent random variables $X_{1}, X_{2}, Y_{1}$, and $Y_{2}$ such that $X_{1} \preceq_{n_{1}-S D} Y_{1}$ and $X_{2} \preceq_{n_{2}-S D} Y_{2}$. Then the two following items (a) and (b) hold for all mixed risk-averse utility functions $u$ from order 1 to $n_{1}+n_{2}$ :

(a) $w\left(x ; Y_{1}+Y_{2}, X_{1}+X_{2}\right) \geq w\left(x ; Y_{1}+Y_{2}, X_{1}+Y_{2}\right)+w\left(x ; Y_{2}+Y_{1}, X_{2}+Y_{1}\right)$

(b) $w\left(x ; Y_{1}+Y_{2}, X_{1}+X_{2}\right) \leq w\left(x ; Y_{1}+X_{2}, X_{1}+X_{2}\right)+w\left(x ; Y_{2}+X_{1}, X_{2}+X_{1}\right)$.

Item (a) was already provided by Ebert et al. (2017) and corresponds to Eq. (15). Item (b) can also be interpreted in the same spirit as Ebert et al. (2017) but in terms of mutual mitigation instead of mutual aggravation as follows: the pain due to the two risk changes at once (passage from $\left(Y_{1}+Y_{2}\right)$ to $\left.\left(X_{1}+X_{2}\right)\right)$ is smaller than the pain due to the aggravation of the first risk plus the pain due to the aggravation of the second risk.

The difference between the two results - mutual mitigation in item (b) of Corollary 4, mutual aggravation in item $(a)$ - is explained by the comparison of the right-hand sides (RHS) in each item. On the RHS of item $(a)$, the decision maker switches twice from a low-risk to a mixed-risk situation. On the RHS of item (b), she switches twice from a mixed-risk to a high-risk situation. This is more painful. This leads therefore to interpret the difference as the outcome of an increasing utility cost of changes in risk. Moving from low-risk to mixed-risk situations is less painful than moving from mixed-risk to high-risk situations.

\section{CONCLUSION}

The paper provides a generalization of non-monetary measures of risk by introducing the concept of $n$ th-order utility premium. This measure reflects the degree of pain due to

\footnotetext{
${ }^{18}$ Indeed, it is easy to verify that $w(x+Z ; Y, X)$ and $w(x ; Y+Z, X+Z)$ both writes as: $E[u(x+Z+$ $Y)]-E[u(x+Z+X)]$.
} 
facing the transition from one risk to a more severe one, with changes in risk expressed through the concept of stochastic dominance of order $n$. The measure is not quite new, since it was used in particular by Eeckhoudt et al. (2009) and by Ebert et al. (2017), without being formally defined. Our objective is to put explicitly the focus on this concept and to analyze systematically some of its key properties. Other non-monetary measures of risk previously defined in the literature, such as the prudence utility premium and the temperance utility premium are special cases of our $n$ th-order utility premium.

We first address the issue of deciding whether it is beneficial to merge changes in risk or not when the $n$ th-order utility premium is used as the decision tool. Our results show that a decision maker whose preferences are mixed risk-averse will feel more pain from merging increases in risk than from facing them in separate entities and we provide an example. We also show that this result implies the property of superadditivity of the $n$ th-order utility premium. We present an illustration in the spirit of Samuelson (1963) who pointed out that risk-averters prefer to subdivide risks instead of facing them in one shot. This holds here for increases in risk and for mixed risk-averse decision makers.

We then turn to analyzing the impact of changes in risk when other risks are in the background and are also subject to changes. This brings us closer to recent contributions in Ebert et al. (2017) emphasizing mutual aggravation of multiple risk changes. Similarly, we use a seminal result in Eeckhoudt et al. (2009) to show that the $n$ th-order utility premium increases when the decision maker faces a riskier wealth under mixed risk aversion. However, our last results emphasize that merging increases in risk may become beneficial if risks in two separate entities are considered as background risks of each other, and if the decision maker is aware that both risks will deteriorate. This means that mutual mitigation of risks is also possible under some circumstances.

The results in this paper provide new interpretations of the alternating signs of higher derivatives of the utility function. As all commonly-used utility functions in economic theory, with the first derivative being positive and the second one being negative, exhibit mixed risk aversion, our results then apply to most individuals facing a deterioration in their risk environment.

Our focus on the properties of the $n$ th-order utility premium represents a modest step in our knowledge of the impact of multiple-risk deteriorations. In particular, we limited our study to the case of additive risks. But not all risks are additive. For instance the hazards to which a property is exposed and the random changes in its value are multiplicative, not additive. The study of the utility premium under $n$ th-order changes of multiplicative risks remains to be addressed. 


\section{APPENDIX}

\section{Proof of Proposition 1.}

We have $X_{2} \preceq_{n_{2}-S D} Y_{2}$ and $Y_{1} \preceq_{2-S D} 0$. Applying Eeckhoudt et al. (2009), we know that $E\left[u\left(x+Y_{2}\right)\right]+E\left[u\left(x+X_{2}+Y_{1}\right)\right]-E\left[u\left(x+X_{2}\right)\right]-E\left[u\left(x+Y_{1}+Y_{2}\right)\right] \leq 0$ for all $u$ such that $(-1)^{(1+k)} u^{(k)} \geq 0 \forall k=1, \ldots, n_{2}+2$.

Analogously, we have $X_{1} \preceq_{n_{1}-S D} Y_{1}$ and $Y_{2} \preceq_{2-S D} 0$. Applying Eeckhoudt et al. (2009), we know that $E\left[u\left(x+Y_{1}\right)\right]+E\left[u\left(x+X_{1}+Y_{2}\right)\right]-E\left[u\left(x+X_{1}\right)\right]-E\left[u\left(x+Y_{1}+Y_{2}\right)\right] \leq 0$ for all $u$ such that $(-1)^{(1+k)} u^{(k)} \geq 0 \forall k=1, \ldots, n_{1}+2$. However, from Eeckhoudt et al. (2009) we get $E\left[u\left(x+Y_{1}+Y_{2}\right)\right]+E\left[u\left(x+X_{1}+X_{2}\right)\right]-E\left[u\left(x+X_{1}+Y_{2}\right)\right]-E\left[u\left(x+Y_{1}+X_{2}\right)\right] \leq 0$ for all $u$ such that $(-1)^{(1+k)} u^{(k)} \geq 0 \forall k=1, \ldots, n_{1}+n_{2}$. Consequently, if $u$ is such that $(-1)^{(1+k)} u^{(k)} \geq 0 \forall k=1, \ldots, n_{1}+n_{2}, \forall n_{1} \geq 2, \forall n_{2} \geq 2$ then the following inequality holds: $\left(E\left[u\left(x+Y_{2}\right)\right]+E\left[u\left(x+X_{2}+Y_{1}\right)\right]-E\left[u\left(x+X_{2}\right)\right]-E\left[u\left(x+Y_{1}+Y_{2}\right)\right]\right)+(E[u(x+$ $\left.\left.\left.Y_{1}\right)\right]+E\left[u\left(x+X_{1}+Y_{2}\right)\right]-E\left[u\left(x+X_{1}\right)\right]-E\left[u\left(x+Y_{1}+Y_{2}\right)\right]\right)+\left(E\left[u\left(x+Y_{1}+Y_{2}\right)\right]+E[u(x+\right.$ $\left.\left.\left.X_{1}+X_{2}\right)\right]-E\left[u\left(x+X_{1}+Y_{2}\right)\right]-E\left[u\left(x+Y_{1}+X_{2}\right)\right]\right) \leq 0$. It rewrites equivalently as: $E[u(x+$ $\left.\left.Y_{1}\right)\right]-E\left[u\left(x+X_{1}\right)\right]+E\left[u\left(x+Y_{2}\right)\right]-E\left[u\left(x+X_{2}\right)\right] \leq E\left[u\left(x+Y_{1}+Y_{2}\right)\right]-E\left[u\left(x+X_{1}+X_{2}\right)\right.$ that is equivalent to $w\left(x ; Y_{1}, X_{1}\right)+w\left(x ; Y_{2}, X_{2}\right) \leq w\left(x ; Y_{1}+Y_{2}, X_{1}+X_{2}\right)$ that ends the proof.

\section{Proof of Corollary 1.}

Let's define $Y=Y_{1}+Y_{2}$. Assuming $X_{1} \preceq_{n_{1}-S D}\left(Y_{1}+Y_{2}\right)$ and $X_{2} \preceq_{n_{2}-S D}\left(Y_{1}+Y_{2}\right)$, we want to prove that Proposition 1 implies Corollary 1. As $Y_{1}, Y_{2}, X_{1}, X_{2}$ are mutually independent, we have $Y \perp X_{1}, Y \perp X_{2}$. As $Y_{1} \preceq_{2-S D} 0$ and $Y_{2} \preceq_{2-S D} 0$, we have (using the convolution property) $Y_{1}+Y_{2} \preceq_{2-S D} 0$. Assumptions of Corollary 1 hold.

Eq. (17) (i.e., the claim of Corollary 1) rewrites then $w\left(x ; Y_{1}+Y_{2}, X_{1}+X_{2}\right) \geq$ $w\left(x ; Y_{1}+Y_{2}, X_{1}\right)+w\left(x ; Y_{1}+Y_{2}, X_{2}\right)$ that we label $\left(17^{\prime}\right)$. Eq. (16) (i.e. the claim of Proposition 1) writes as $w\left(x ; Y_{1}+Y_{2}, X_{1}+X_{2}\right) \geq w\left(x ; Y_{1}, X_{1}\right)+w\left(x ; Y_{2}, X_{2}\right)$.

We show (see proof below) that $w\left(x ; Y_{1}, X_{1}\right) \geq w\left(x ; Y_{1}+Y_{2}, X_{1}\right)$ and $w\left(x ; Y_{2}, X_{2}\right) \geq$ $w\left(x ; Y_{1}+Y_{2}, X_{2}\right)$. Using this result we obtain Eq. $(16) \Rightarrow$ Eq. $\left(17^{\prime}\right)$.

To prove that $w\left(x ; Y_{1}, X_{1}\right) \geq w\left(x ; Y_{1}+Y_{2}, X_{1}\right)$, we use the definition of the $n$ thorder utility premium. Then $w\left(x ; Y_{1}, X_{1}\right) \geq w\left(x ; Y_{1}+Y_{2}, X_{1}\right)$ writes equivalently as $E\left[u\left(x+Y_{1}+Y_{2}\right)\right] \leq E\left[u\left(x+Y_{1}\right)\right]$ which is true for all $u$ such that $u^{\prime \prime}<0$ as $Y_{1}$ and $Y_{2}$ are zero-mean independent risks. The proof is the same for $w\left(x ; Y_{2}, X_{2}\right) \geq w\left(x ; Y_{1}+Y_{2}, X_{2}\right)$.

\section{Proof of Corollary 2.}

Using the definition of $w, w\left(x+X_{2} ; Y, X\right)-w\left(x+Y_{2} ; Y, X\right) \geq 0$ rewrites equivalently as $E\left[u\left(x+Y+X_{2}\right)\right]-E\left[u\left(x+X+X_{2}\right)\right] \geq E\left[u\left(x+Y+Y_{2}\right)\right]-E\left[u\left(x+X+Y_{2}\right)\right]$, that is equivalent to $E\left[u\left(x+Y+X_{2}\right)\right]+E\left[u\left(x+X+Y_{2}\right)\right] \geq E\left[u\left(x+Y+Y_{2}\right)\right]+E\left[u\left(x+X+X_{2}\right)\right]$. Following Eeckhoudt et al. (2009), this last expression is equivalent to $(-1)^{(1+k)} u^{(k)} \geq 0$ 
for $k=1, \ldots, n_{1}+n_{2}$.

\section{REFERENCES}

Arrow, K. (1970). Essays in the Theory of Risk Bearing, Amsterdam: North-Holland.

Artzner, P., Delbaen, F., Eber, J.M. and Heath, D. (1999). 'Coherent risk measures', Mathematical Finance, 9, 203-228.

Brockett, P.L., and Golden, L.L. (1987). 'A class of utility functions containing all the common utility functions'. Management Science, 33(8), 955-964.

Bühlmann, H. (1985). 'Premium calculation from top down', ASTIN Bulletin, 15(2), 89-101.

Caballé, J. and Pomansky, A. (1996). 'Mixed risk aversion', Journal of Economic Theory, 71, 485-513.

Courbage, C. and Rey, B. (2010). 'On non monetary measures in the face of risks and the signs of the derivatives', Bulletin of Economic Research, 69(3), 295-304.

Crainich, D. and Eeckhoudt, L. (2008). 'On the intensity of downside risk aversion', Journal of Risk and Uncertainty, 36, 267-276.

Deck, G. and Schlesinger, H. (2010). 'Exploring higher-order risk effects', Review of Economic Studies, 77, 1403-1420.

Deck, G. and Schlesinger, H. (2014). 'Consistency of higher-order risk preferences', Econometrica, 82, 1913-1943.

Ebert, S. (2013). 'Moment characterization of higher-order risk preferences', Theory and Decision, 74, 267-284.

Ebert, S., Nocetti, D.C. and Schlesinger, H. (2017). 'Greater mutual aggravation', forthcoming in Management Science.

Eeckhoudt, L. and Gollier, C. (2001). 'Which shape for the cost curve of risk?', Journal of Risk and Insurance, 68(3), 387-402.

Eeckhoudt, L. and Gollier, C. (2013). 'The effects of changes in risk on risk taking: a survey', Handbook of Insurance, Dionne G. (ed), Kluwer Academic Publisher, 123-135.

Eeckhoudt, L., Rey, B. and Schlesinger, H. (2007). 'A good sign for multivariate risk taking', Management Science, 53, 117-124.

Eeckhoudt, L. and Schlesinger, H. (2006). 'Putting risk in its proper place', American Economic Review, 96, 280-289.

Eeckhoudt, L. and Schlesinger, H. (2009). 'On the utility premium of Friedman and Savage', Economics Letters, 105(1), 46-48.

Eeckhoudt, L., Schlesinger, H., and Tsetlin, E. (2009). 'Apportioning risks via stochastic dominance', Journal of Economic Theory, 113, 1-31.

Ekern, S. (1980). 'Increasing Nth degree risk', Economics Letters, 6, 329-333. 
Friedman, M. and Savage, L.J. (1948). 'The utility analysis of choices involving risk', Journal of Political Economy, 56, 279-304.

Gerber, H.U. and Goovaerts, M.J. (1981). 'On the representation of additive principles of premium calculation', Scandinavian Actuarial Journal, 4, 221-227

Gollier, C. and Pratt, J.W. (1996). 'Risk vulnerability and the tempering effect of background risk', Econometrica, 64, 1109-1123.

Huang, J. and Stapleton, R. (2015) 'The utility premium of Friedman and Savage, comparative risk aversion and comparative prudence', Economics Letters, 134, 34-36.

Ingersoll, J.F. (1987). Theory of Financial Decision Making, Rowman \& Littlefield, Lanham, MD.

Jean, W.H. (1980). 'The geometric mean and stochastic dominance', Journal of Finance, $35,151-158$.

Jean, W.H. (1984). 'The harmonic mean and other necessary conditions for stochastic dominance', Journal of Finance, 39, 527-534.

Kimball, M.S. (1990). 'Precautionary saving in the small and in the large', Econometrica, 58, 53-73.

Kimball, M.S. (1992). 'Precautionary motives for holding assets', The New Palgrave Dictionary of Money and Finance, London: Macmillan, 3, 158-161.

Lajeri-Chaherli, F. (2004). 'Proper prudence, standard prudence and precautionary vulnerability', Economics Letters, 82, 29-34.

Li, J. and Liu, L. (2014) 'The monetary utility premium and interpersonal comparisons', Economics Letters, 125, 257-260.

Liu, L. (2014) 'Precautionary saving in the large: nth degree deterioration in future income' Journal of Mathematical Economics, 59, 169-172.

Menegatti, M. (2011). 'The risk premium and the effects of risk on agents utility', Research in Economics, 65, 89-94.

Menegatti, M. (2015). 'New results on high-order risk changes', European Journal of Operational Research, 243, 678-681.

Menezes, C., Geiss, C. and Tressler, J. (1980) 'Increasing downside risk', American Economic Review, 70, 921-932.

Pratt, J.W. (1964). 'Risk aversion in the small and in the large', Econometrica, 32, 122-136.

Pratt, J.W. (1988). 'Aversion to one risk in the presence of others', Journal of Risk and Uncertainty, 1, 395-413.

Roger, P. (2011). 'Mixed risk aversion and preference for risk disaggregation: A story of moments', Theory and Decision, 70, 27-44.

Rothschild, M. and Stiglitz, J. (1970) 'Increasing risk: I. A definition', Journal of Economic Theory, 2, 225-243. 
Samuelson, P. (1963). 'Risk and uncertainty: A fallacy of large numbers', Sciencia, 6 th series, $57,1-6$.

\section{ACKNOWLEDGMENTS}

We would like to thank participants in the CEAR/MRIC Behavioral Insurance Workshop 2016 in Munich and in the 44th Seminar of the European Group of Risk and Insurance Economists in London as well as two anonymous referees for valuable comments and discussions. We are also very grateful to Sebastian Ebert for his careful reading of successive versions of this paper. His appropriate comments have substantially improved the paper. 